\title{
Rotavirus antigen, cytokine, and neutralising antibody profiles in sera of children with and without HIV infection in Blantyre, Malawi
}

\author{
Jennifer J. Hull ${ }^{1}$, Nigel Cunliffe ${ }^{2}$, Khuzwayo C. Jere ${ }^{2,3}$, Sung-Sil Moon ${ }^{1}$, Yuhuan Wang ${ }^{1}$, \\ Umesh Parashar ${ }^{1}$, Baoming Jiang ${ }^{1}$
}

1. Division of Viral Diseases, Centers for Disease Control and Prevention, Atlanta, Georgia, USA

2. Department of Clinical Infection, Microbiology and Immunology; University of Liverpool, Liverpool, United Kingdom

3. Malawi-Liverpool-Wellcome Trust Clinical Research Programme, College of Medicine, University of Malawi, Blantyre, Malawi

Correspondence: Dr Baoming Jiang (bxj4@cdc.gov)

\section{Background}

\section{Abstract}

Rotavirus and HIV infection are major causes of death among children in sub-Saharan Africa. A previous study reported no association between concomitant HIV infection and rotavirus disease severity among hospitalised children in Malawi. This study examined rotavirus antigenaemia and broader immune responses among HIV-infected and uninfected children.

Methods

Stored $\left(-80^{\circ} \mathrm{C}\right)$, paired sera from acute and convalescent phases of Malawian children less than 5 years old, hospitalised for acute gastroenteritis in the primary study, collected from July 1997 to June 1999, were utilised. Among children older than 15 months, HIV infection was defined as the presence of HIV antibody in the blood, when confirmed by at least 2 established methods. For those younger than 15 months, nested polymerase chain reaction (PCR) amplification of proviral DNA was used for verification. All were followed for up to 4 weeks after hospital discharge. Rotavirus antigen levels in sera were measured with Premier ${ }^{\mathrm{TM}}$ Rotaclone ${ }^{\circledR}$ rotavirus enzyme immunoassay (EIA) kit. Acute-phase sera were examined for 17 cytokines, using Luminex fluorescent bead human cytokine immunoassay kit. Rotavirus-specific IgA and neutralising activity were determined by EIA and microneutralisation (MN) assay, respectively. Human strains and bovine-human reassortants were propagated in MA104 cells with serum-free Iscove's Modified Dulbecco's Medium (IMDM). Differences in results, from specimens with and without HIV infection, were analysed for statistical significance using the chi-square test.

Results

We detected rotavirus antigen in 30\% of the HIV-infected and 21\% HIV-uninfected, in the acute-phase sera. HIV-infected children developed slightly prolonged rotavirus antigenaemia compared to HIV-uninfected children.

Conclusions

Rotavirus-specific IgA seroconversion rates and neutralising titres were similar in HIV-infected and HIV-uninfected children, thus, HIV infection had no major effect on immune responses to rotavirus infection.

\section{Introduction}

Group A rotavirus infection is an important cause of paediatric hospitalisation due to severe diarrhoea, and thus is a global public health concern. Although rotavirus (RV) is ubiquitous and incidence of rotavirus is similar among children in industrial and developing countries, very few deaths occur in affluent countries, whereas most rotavirusrelated deaths are in the developing countries of Asia and Africa, with an estimated 243.3 deaths per 100,000 children under 5 years of age, annually. ${ }^{1}$ Similarly, human immunodeficiency virus (HIV) infection and acquired immune deficiency syndrome (AIDS) remain among the most significant public health challenges in these settings, with about 3.2 million children under 14 years of age infected with HIV worldwide; approximately $90 \%$ of them are in sub-Saharan Africa. ${ }^{2}$

The high burden of rotavirus-related diarrhoea occurring among children under 5 years old makes vaccination a priority. ${ }^{3}$ Two live oral rotavirus vaccines, Rotarix (GlaxoSmithKline, human monovalent G1[P8]), and RotaTeq (Merck, pentavalent human-bovine reassortant, G1, G2, G3, and G4, and P[8]) have been recommended by the World Health Organization (WHO) as a part of the routine programme of childhood immunisation. ${ }^{4}$ Studies have revealed high vaccine efficacy in developed countries, but aberrantly lower results in developing countries. ${ }^{5,6}$ Reasons for lower efficacy in low-income countries are not completely understood, but suspected factors include maternal antibodies in serum, neutralising antibodies in breastmilk, concurrent oral polio vaccine administration, malnutrition, strain diversity and coinfections with other pathogens.

Rotavirus morbidity and mortality in children could potentially be exaggerated by HIV infection, as HIV is associated with progressive reduction in CD4 cell count, chronic immune activation, and altered levels of circulating cytokines. ${ }^{8}$ However, studies have shown that children in countries of Asia and sub-Saharan Africa, with high rotavirus gastroenteritis-associated fatality rates and with concomitant HIV infection, recover from acute rotavirus infections. ${ }^{9,10}$ These results paved the way for the WHO recommendation that rotavirus vaccines be incorporated into all national immunisation programmes. ${ }^{11}$

Rotavirus infection, previously thought to be localised in the epithelial cells of the lining of the small intestine, has been detected in the blood, body fluids, and tissues, suggesting the possibility that rotavirus escapes the intestine into the circulatory system. ${ }^{12,13}$ The primary study examined the effect of concomitant HIV infection on rotavirus gastroenteritis in hospitalised Malawian children and found no differences in rotavirus severity for those with and without HIV infection. Equally, these children recovered from acute rotavirus infections. ${ }^{10}$

In this extended study, we assessed whether rotavirus antigen was present in sera, and systematically investigated the possible influence of HIV infection on rotavirus pathogenesis and immunity among children in Malawi, by employing a broader repertoire of rotavirus tests for rotavirus antigen, circulating cytokines, immunoglobulin A ( $\operatorname{Ig} A)$, and neutralising activity. 
Table 1: Comparison of demographic and rotavirus antigenaemia data by HIV status among children with rotavirus diarrhoea in Blantyre, Malawi

\begin{tabular}{|c|c|c|c|c|c|}
\hline \multirow{2}{*}{ Variables } & \multicolumn{2}{|c|}{ HIV-infected (N = 33) } & \multicolumn{2}{|c|}{ HIV-uninfected $(\mathrm{N}=42)$} & \multirow{2}{*}{ P-value } \\
\hline & Frequency (\%) & Median (Range) & Frequency (\%) & Median (Range) & \\
\hline \multicolumn{6}{|l|}{ Age } \\
\hline 0-6 months & $18(55 \%)$ & $4.5(1-6)$ & $23(55 \%)$ & $4.0(2-6)$ & 0.97 \\
\hline$>6$ months & $15(45 \%)$ & $9.0(7-17)$ & $19(45 \%)$ & $10.0(7-21)$ & 0.96 \\
\hline \multicolumn{6}{|l|}{ Gender } \\
\hline Male & $15(45 \%)$ & - & $23(55 \%)$ & - & 0.32 \\
\hline Female & $18(55 \%)$ & - & $19(45 \%)$ & - & 0.33 \\
\hline \multicolumn{6}{|l|}{$\begin{array}{l}\text { Rotavirus } \\
\text { antigenaemia }\end{array}$} \\
\hline Acute phase & $10(30 \%)$ & $0.10(0.04-2.11)^{*}$ & $9(21 \%)$ & $0.07(0.04-1.26)^{*}$ & 0.11 \\
\hline $\begin{array}{c}\text { Convalescent } \\
\text { phase }\end{array}$ & $3(9 \%)$ & $0.10(0.04-0.33)^{*}$ & $1(2 \%)$ & $0.05(0.04-0.15)^{*}$ & 0.16 \\
\hline
\end{tabular}
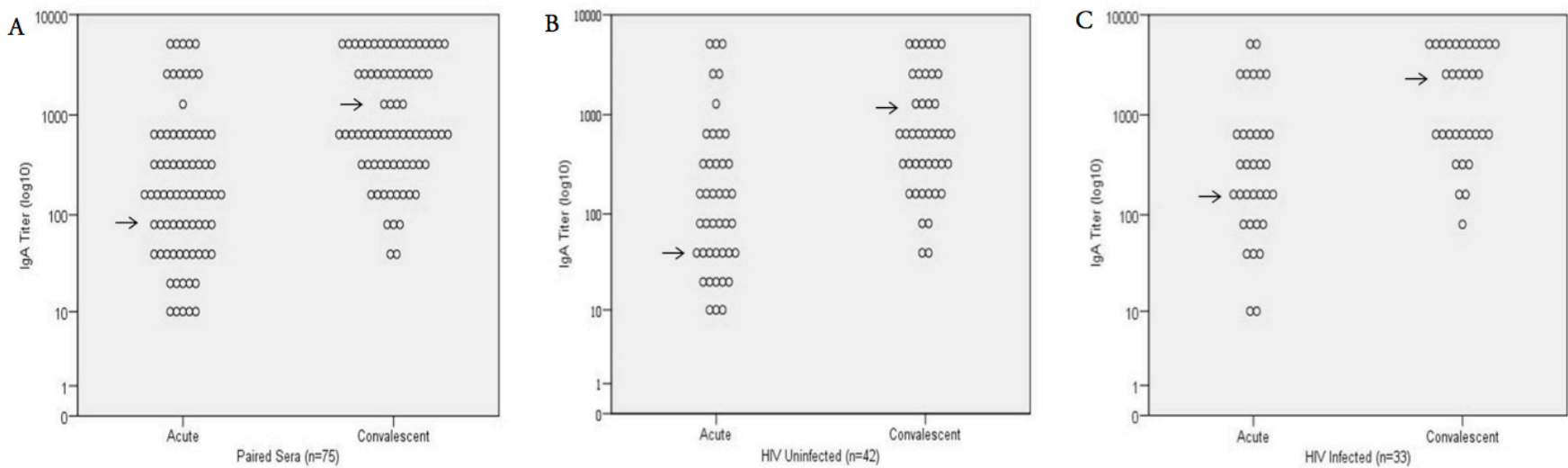

Figure 1: Rotavirus-specific IgA titres in acute- and convalescent-phase sera of children, Malawi

Paired sera from (A) total cohort, (B) HIV-infected, and (C) HIV-uninfected were tested for rotavirus IgA as described in the text. Median titre is indicated using a black arrow.

\section{Methods}

Stored $\left(-80^{\circ} \mathrm{C}\right)$, paired sera from acute and convalescent phases of Malawian children under 5 years old, hospitalised for acute gastroenteritis in the primary study, collected from July 1997 to June 1999, were utilised. Children with and without HIV infection were verified. Briefly, among children older than 15 months, HIV infection was defined as the presence of HIV antibody in the blood, when confirmed by at least 2 established methods. For those younger than 15 months, nested polymerase chain reaction (PCR) amplification of proviral DNA was used for verification; both previously described. ${ }^{10}$ All were followed for up to 4 weeks after hospital discharge. Rotavirus antigen levels in sera were measured with Premier ${ }^{\mathrm{TM}}$ Rotaclone ${ }^{\circledR}$ Rotavirus enzyme immunoassay (EIA) kit (Meridian Diagnostics, Cincinnati, $\mathrm{OH}$, USA), according to manufacturer's instructions. Acute-phase sera were examined for 17 cytokines, using Luminex fluorescent bead human cytokine immunoassay kit (Bio-Plex Pro ${ }^{\mathrm{TM}}$ Assays; Bio-Rad Laboratories, Hercules, CA, USA), according to manufacturer's instructions. The cytokines examined were IL-1 $\beta$, IL-2, IL-7, IL-12, IL17, IFN- $\gamma$, TNF- $\alpha$, IL-4, IL-5, IL-6, IL-10, IL-13, IL-8, G-CSF, GM-CSF, MCP-1(MCAF), and MIP-1 $\beta$. Rotavirus- specific IgA and neutralising activity were determined by EIA and microneutralisation (MN) assay, respectively. ${ }^{14}$ Human strains: Rotarix (G1P[8]), MW333 (G8P[4]) and WI61 (G9P[8]); bovine WC3 (G6P[5]); and bovine-human reassortants RotaTeq WC3 × WI79-9 (G1P[5]) and WC3 $\times$ W179-4 (G6P[8]) were propagated in MA104 cells with serum-free Iscove's Modified Dulbecco's Medium (IMDM). Differences in results, from specimens with and without HIV infection, were analysed for statistical significance using the chi-square test.

In the primary study, $6 \mathrm{HIV}$-positive and $5 \mathrm{HIV}$-negative samples were excluded in the analysis for reasons of late onset of illness. ${ }^{10}$ However, we were unable to find original records and thus included all samples, with sufficient volume, in the present study.

\section{Ethical considerations}

The Malawi National Health Sciences Research Committee (NHSRC) gave ethical approval and informed consent was obtained as documented in the parent study. ${ }^{10}$ Specimens and data were coded with unique identifiers for anonymity, and given to the CDC (RD 2010 6007) under a non-disclosure agreement which unequivocally prohibits the release of personally identifiable information about participants. 
Table 2: Comparison of serology data by HIV status among children with rotavirus diarrhoea in Blantyre, Malawi

\begin{tabular}{|c|c|c|c|c|c|}
\hline \multirow{2}{*}{ Variables } & \multicolumn{2}{|c|}{ HIV-infected } & \multicolumn{2}{|c|}{ HIV-uninfected } & \multirow{2}{*}{ P-value } \\
\hline & Frequency $(\%)$ & Median (Range) & Frequency (\%) & Median (Range) & \\
\hline IgA titre & $\mathrm{N}=33$ & & $\mathrm{~N}=42$ & & \\
\hline Acute phase & & $320(10-5120)$ & & $160(10-5120)$ & 0.48 \\
\hline Convalescent phase & & $2560(80-5120)$ & & $640(40-5120)$ & 0.15 \\
\hline Seroconversion* & $18(51 \%)$ & & $21(50 \%)$ & & 0.24 \\
\hline Microneutralisation titre & $\mathbf{N}=32$ & & $\mathrm{~N}=40$ & & \\
\hline \multicolumn{6}{|l|}{ Human Rotarix G1P8 } \\
\hline Acute phase & & $80(1-1280)$ & & $80(1-160)$ & 0.81 \\
\hline Convalescent phase & & $160(40-2560)$ & & $160(40-1280)$ & 0.53 \\
\hline Seroconversion* & $10(31 \%)$ & & $10(25 \%)$ & & \\
\hline \multicolumn{6}{|l|}{ Human MW333 G8P4 } \\
\hline Acute phase & & $20(1-640)$ & & $20(1-640)$ & 0.61 \\
\hline Convalescent phase & & $60(1-2560)$ & & $40(1-2560)$ & 0.79 \\
\hline Seroconversion* & $14(44 \%)$ & & $21(50 \%)$ & & \\
\hline \multicolumn{6}{|l|}{ Human W161 G9P8 } \\
\hline Acute phase & & $20(1-10,240)$ & & $20(1-320)$ & 0.81 \\
\hline Convalescent phase & & $80(-2560)$ & & $160(1-10,240)$ & 0.91 \\
\hline Seroconversion* & $19(59 \%)$ & & $27(68 \%)$ & & \\
\hline \multicolumn{6}{|l|}{$\begin{array}{l}\text { Human-bovine } \\
\text { reassortants }\end{array}$} \\
\hline \multicolumn{6}{|l|}{-RotaTeq G1P5 } \\
\hline Acute phase & & $1(1-40)$ & & $1(1-40)$ & 0.93 \\
\hline Convalescent phase & & $20(1-160)$ & & $20(1-320)$ & 0.56 \\
\hline Seroconversion* & $6(19 \%)$ & & $6(15 \%)$ & & \\
\hline \multicolumn{6}{|l|}{- RotaTeq G6P8 } \\
\hline Acute phase & & $1(1-160)$ & & $1(1-40)$ & 0.57 \\
\hline Convalescent phase & & $1(1-1280)$ & & $1(1-160)$ & 0.44 \\
\hline Seroconversion* & $6(19 \%)$ & & $3(8 \%)$ & & \\
\hline \multicolumn{6}{|l|}{ Bovine WC3 G6P5 } \\
\hline Acute phase & & $1(1-320)$ & & $1(1-40)$ & 0.57 \\
\hline Convalescent phase & & $1(1-160)$ & & $1(1-80)$ & 0.60 \\
\hline Seroconversion* & $4(13 \%)$ & & $3(8 \%)$ & & \\
\hline
\end{tabular}

\section{Results and Discussion}

Paired sera, with sufficient volume, were selected from 33 HIV-positive and $42 \mathrm{HIV}$-negative children who completed the follow-up, displaying comparable demographic variables among children with or without HIV infection (Table 1). Rotavirus antigen was detected in $19(25 \%)$ of 75 acutephase serum samples. This rate was comparable to a report in the United States, but was lower than the rates of $43 \%$ to $76 \%$ reported in Jamaica, Finland, and Japan. ${ }^{13,15,16}$

However, in Malawi, rotavirus antigenaemia was slightly more prevalent among HIV-infected than the uninfected children $(30 \%$ versus $21 \%, \mathrm{P}=0.11)$ in the acute phase and similarly, in the convalescent phase $(9 \%$ and $2 \%, \mathrm{P}=0.16)$, respectively. Similar reports were observed among HIVinfected children in Kenya. ${ }^{17}$ Our detection of rotavirus antigen, in the convalescent phase of both populations, 3 to 4 weeks after hospitalisation, is unlike other reports, where levels peaked 1 to 3 days after the onset of acute rotavirus gastroenteritis and were undetectable beyond a week. ${ }^{15,18}$ Parallel results were also observed in paired plasma specimens from this collection (data not shown), validating our results obtained from sera. Our finding of the prolonged rotavirus antigenaemia in HIV-infected children mimics the prolonged rotavirus antigen shedding in stool of children from the original study. ${ }^{10}$

To examine whether HIV infection influenced host immune response, we analysed paired sera for rotavirus-specific IgA (Table 2). Although median IgA titres were higher in the HIV-infected than the HIV-uninfected children, for both the acute (320 vs 160) and convalescent (2560 vs 640) phases, similar seroconversion rates $(51 \%$ vs $50 \%$ ) were observed in the 2 populations, respectively. Our seroconversion rates were lower than that $(76 \%)$ in the primary study, ${ }^{10}$ possibly due to sample storage, difference in reagents, assay design, and analysis methods. 
From 1997 to 1999 , diverse rotavirus strains, predominantly G1P[8], G8P[6], G3P[8], G8P[4] and G4P[8], were present in Malawi. ${ }^{19}$ We therefore, analysed 72 paired serum samples for neutralising activity against 3 human (Rotarix G1P[8], MW333 G8P[4] and WI61 G9P[8]), 1 bovine (WC3 G6P[5]) and 2 bovine-human reassortant (RotaTeq G1P[5] and G6P[8]) rotavirus strains, using MN assay (Table 2). We found that children with natural infection developed high titres of cross-reactive neutralising activity responses against homotypic and heterotypic human strains. By contrast, children developed little or no neutralising activity to bovine or bovine-human reassortant strains. However, we observed no significant differences in median titres of neutralising activity against both human and animal strains in the acute and convalescent phases between the HIV-infected and HIVuninfected population.

To examine if immune status has any effect on host response to rotavirus infection, we analysed blood from HIV-infected children who developed AIDS (CD4 count $<500 / \mu \mathrm{L})$ compared to those who did not develop AIDS (CD4 count $\geq 500 /$ $\mu \mathrm{L})$, (Table 3). The sample size with available CD4 count data was small; nonetheless, there seemed to be no difference in antigenaemia between AIDS patients and

Table 3: Comparison of rotavirus antigenaemia and IgA titres in children with rotavirus diarrhoea in Blantyre, Malawi, according to HIV status and CD4 count

\begin{tabular}{|c|c|c|c|}
\hline \multirow{2}{*}{ Variables } & \multicolumn{2}{|c|}{ HIV-infected } & \multirow{2}{*}{$\begin{array}{c}\text { HIV-uninfected }(\mathrm{N}=42) \\
\mathrm{CD} 4 \text { count } \geq 500 / \mu \mathrm{L}\end{array}$} \\
\hline & $\mathrm{CD} 4$ count $<500 / \mu \mathrm{L}$ & $\mathrm{CD} 4$ count $\geq 500 / \mu \mathrm{L}$ & \\
\hline Rotavirus antigenaemia & $\mathbf{N}=4$ & $N=5$ & $\mathbf{N}=9$ \\
\hline Acute phase & $2(50 \%)$ & $3(60 \%)$ & $2(22 \%)$ \\
\hline Convalescent phase & $1(25 \%)$ & $1(20 \%)$ & $1(11 \%)$ \\
\hline IgA geometric mean titre & $\mathbf{N}=\mathbf{4}$ & $N=5$ & $\mathbf{N}=9$ \\
\hline Acute phase* & $433(80-2560)$ & $279(10-2560)$ & $202(20-2560)$ \\
\hline Convalescent phase* & $538(160-2560)$ & $845(320-2560)$ & $871(160-2560)$ \\
\hline $\begin{array}{l}\text { Fold-change from acute to } \\
\text { convalescent phase }\end{array}$ & 1 & 3 & 4 \\
\hline
\end{tabular}

*denotes IgA geometric mean titre (range) in acute- and convalescent-phase sera

Table 4: Profiles of cytokines in sera of children with acute rotavirus diarrhoea in Blantyre, Malawi, by HIV status

\begin{tabular}{|c|c|c|c|c|c|c|}
\hline \multirow{2}{*}{ Cytokines } & \multicolumn{2}{|c|}{ Paired sera $(N=40)$} & \multicolumn{2}{|c|}{ HIV-infected (N = 20) } & \multicolumn{2}{|c|}{ HIV-uninfected $(\mathrm{N}=20)$} \\
\hline & Range & Median & Range & Median & Range & Median \\
\hline \multicolumn{7}{|l|}{ Pro-inflammatory } \\
\hline IL- $1 \beta$ & $2-75$ & 3 & $2-75$ & 3 & 0 & 0 \\
\hline IL-2 & $1-455$ & 25 & $1-455$ & 12 & $1-139$ & 32 \\
\hline IL-7 & $1-83$ & 1 & $1-83$ & 2.5 & $1-21$ & 1 \\
\hline IL-12 & $1-419$ & 1 & $1-419$ & 1 & $1-118$ & 1 \\
\hline IL-17 & $1-70$ & 1 & $1-70$ & 1 & $1-27$ & 2.5 \\
\hline IFN-Y & $1-14,112$ & 252 & $1-14,112$ & 233 & $1-1820$ & 304.5 \\
\hline TNF- $\alpha$ & $1-150$ & 1 & $1-150$ & 1 & $1-8$ & 1 \\
\hline \multicolumn{7}{|l|}{ Anti-inflammatory } \\
\hline IL-4 & $1-13$ & 1 & $1-13$ & 1 & $1-6$ & 1 \\
\hline IL-5 & $0-30$ & 1 & $0-30$ & 1 & $0-3$ & 1 \\
\hline IL-6 & $1-673$ & 58.5 & $1-673$ & 74.5 & $1-322$ & 56 \\
\hline IL-10 & $3-415$ & 21.5 & $5-415$ & 19 & $3-132$ & 25 \\
\hline IL-13 & $4-72$ & 5 & $4-72$ & 5 & $4-9$ & 5 \\
\hline \multicolumn{7}{|l|}{ Chemokines } \\
\hline IL-8 & $1-477$ & 9 & $1-388$ & 14 & $1-477$ & 1.5 \\
\hline G-CSF & $1-375$ & 7.5 & $1-375$ & 17 & $1-78$ & 1 \\
\hline GM-CSF & $1-1667$ & 678 & $1-1343$ & 576 & $1-1667$ & 856.5 \\
\hline MCP-1(MCAF) & $1-191$ & 74.5 & $1-191$ & 60 & $1-140$ & 81 \\
\hline MIP-1 $\beta$ & $78-1411$ & 260 & $78-1411$ & 255.5 & $167-1397$ & 295.5 \\
\hline
\end{tabular}

HIV-infected patients without AIDS; higher antigenaemia was seen in the acute phase of both immunocompromised and non-immunocompromised patients with HIV than in the respective convalescent phases. Our results would suggest that, overall, HIV-positive children with diarrhoea were more likely than their counterparts without HIV infection to have rotavirus in their blood. ${ }^{17}$ Additionally, there was no change in the $\operatorname{Ig} \mathrm{A}$ antibody response in the sera of AIDS patients, from the acute to the convalescent phase (geometric mean titres [GMT] 453 to 538). In contrast, there was a threefold rise in IgA GMT (279 to 845) in HIV-infected patients without AIDS, which was similar to a four-fold rise (202 to 871 ) in the HIV-uninfected children.
Lastly, we examined a random subset of acute sera with sufficient remaining volume, from $20 \mathrm{HIV}$-infected and 20 HIV-uninfected children, for profiles of 17 cytokines (Table 4). A host typically responds to a viral infection by developing innate and adaptive immunity, including production of cytokines and antibodies. ${ }^{20}$ Similarly, rotavirus-activated CD8+ cytotoxic T cells have been shown to be involved in rotavirus immunity. ${ }^{20}$ In contrast, HIV infection has been associated with progressive reduction in CD4 cell count, chronic immune activation, and altered levels of circulating cytokines. ${ }^{8}$ In this study, we observed little or no cytokine differences between the HIV-infected and HIV-uninfected children. In addition, we were able to detect levels of 
several chemokines and a few pro-inflammatory and antiinflammatory cytokines in the early stages of rotavirus diarrhoea. These findings suggest that chemokines may play a pivotal role in both inflammatory and immune responses to rotavirus infection. ${ }^{9}$ Accelerant, pro-inflammatory cytokines, like IL- $1 \beta$ and TNF- $\alpha$, which tend to make diseases worse by producing fever, inflammation, tissue destruction, shock, and even death, ${ }^{21}$ were detected at low levels in a few subjects of this study, corroborating our observations of overall severe disease (severity score $\geq 11$ ) in only $16 \%$ of the study sample and high fever (temperature $\geq 39^{\circ} \mathrm{C}$ ) in $19 \%$ of the children. On the other hand, as elevated levels of IL- 6 and IFN- $\gamma$ were reported in children with natural rotavirus infection in Bangladesh and the United States, ${ }^{22,23}$ we also detected levels of both pro- and anti-inflammatory cytokines (IL-2, IFN- $\alpha$, IL-6, and IL-10) among Malawian children with rotavirus diarrhoea, regardless of HIV status. In general, similar levels of the 17 cytokines tested were observed in children, regardless of their HIV status, and differences between the groups were not significant.

Although the integrity of our collection of serum samples, from the 1997 to 1999 seasons in Malawi, could have been compromised due to collection handling, transportation rigors, and long storage time, our findings, in general, were similar to those obtained in the primary study, which was reported in $2001 .{ }^{10}$ Our sample size was small, and paired sera were not available for all children in the study. In addition, because of the lack of adequate volumes in these old samples, we could not perform all necessary tests, including being able to normalise the rotavirus-specific IgA levels.

Our analysis found that children infected with HIV appeared to be slightly more likely to develop rotavirus antigenaemia and to have longer duration of antigenaemia than the HIV-uninfected children. However, regardless of HIV status, children mounted equivalent cytokine and antibody responses to rotavirus infection. Overall, our findings that HIV infection had no major effect on rotavirus infection and immune response, as determined by rotavirus antigenaemia, cytokines, and neutralising antibodies among children in Malawi, support the recommendation that children with HIV-infection in low-income settings can receive liveattenuated oral rotavirus vaccine, as part of the routine national immunisation programme, to prevent severe dehydrating diarrhoea and deaths.

\section{Acknowledgements}

We wish to thank Drs Jon Gentsch, Houping Wang, Ken Sugata, and Jeffrey Priest for their contributions in this study.

\section{Competing interests}

The authors declare that they have no conflicts of interest.

\section{References}

1. Sanchez-Padilla E, Grais RF, Guerin PJ, Steele AD, Burny ME, Luquero FJ. Burden of disease and circulating serotypes of rotavirus infection in sub-Saharan Africa: systematic review and meta-analysis. The Lancet Infectious diseases.2009;9:567-576.

2. UNAIDS. Global Report- UNAIDS report on the global AIDS epidemic 2013. . Geneva, 2013.
3. Dennehy PH. Rotavirus vaccines: an overview. Clinical microbiology reviews.2008;21:198-208.

4. WHO. Weekly epidemiological record. Rotavirus vaccines: an update, 2009:p. 553-537.

5. Vesikari T, Matson DO, Dennehy P, Van Damme P, Santosham M, Rodriguez Z, et al. Safety and efficacy of a pentavalent human-bovine (WC3) reassortant rotavirus vaccine. The New England journal of medicine.2006;354:23-33.

6. Patel M, Pedreira C, De Oliveira LH, Tate J, Orozco M, Mercado J, et al. Association between pentavalent rotavirus vaccine and severe rotavirus diarrhoea among children in Nicaragua. Jama.2009;301:2243-2251.

7. Moon SS, Wang Y, Shane AL, Nguyen T, Ray P, Dennehy P, et al. Inhibitory effect of breast milk on infectivity of live oral rotavirus vaccines. The Pediatric infectious disease journal.2010;29:919-923.

8. Kedzierska K, Crowe SM. Cytokines and HIV-1: interactions and clinical implications. Antiviral chemistry \& chemotherapy.2001;12:133-150.

9. Steele AD, Cunliffe N, Tumbo J, Madhi SA, De Vos B, Bouckenooghe A. A review of rotavirus infection in and vaccination of human immunodeficiency virus-infected children. J Infect Dis.2009;200 Suppl 1:S57-62.

10. Cunliffe NA, Gondwe JS, Kirkwood CD, Graham SM, Nhlane NM, Thindwa BD, et al. Effect of concomitant HIV infection on presentation and outcome of rotavirus gastroenteritis in Malawian children. Lancet.2001;358:550-555.

11. WHO. Weekly epidemiological record. Rotavirus vaccines WHO position paper, 2013:p. 49-64.

12. Blutt SE, Conner ME. Rotavirus: to the gut and beyond! Current opinion in gastroenterology.2007;23:39-43.

13. Sugata K, Taniguchi K, Yui A, Miyake F, Suga S, Asano Y, et al. Analysis of rotavirus antigenaemia and extraintestinal manifestations in children with rotavirus gastroenteritis. Pediatrics.2008;122:392-397.

14. Groome MJ, Moon SS, Velasquez D, Jones S, Koen A, van Niekerk $N$, et al. Effect of breastfeeding on immunogenicity of oral live-attenuated human rotavirus vaccine: a randomized trial in HIVuninfected infants in Soweto, South Africa. Bulletin of the World Health Organization.2014;92:238-245.

15. Fischer TK, Ashley D, Kerin T, Reynolds-Hedmann E, Gentsch J, Widdowson MA, et al. Rotavirus antigenaemia in patients with acute gastroenteritis. Journal of Infectious Diseases.2005;192:913-919.

16. Hemming M, Huhti L, Rasanen S, Salminen M, Vesikari T. Rotavirus antigenaemia in children is associated with more severe clinical manifestations of acute gastroenteritis. The Pediatric infectious disease journal.2014;33:366-371.

17. Kiulia NM, Nyaundi JK, Peenze I, Nyachieo A, Musoke RN, Steele $\mathrm{AD}$, et al. Rotavirus infections among HIV-infected children in Nairobi, Kenya. Journal of tropical pediatrics.2009;55:318-323.

18. Chiappini E, Galli L, de Martino M. Viremia and clinical manifestations in children with rotavirus infection. The Journal of infectious diseases.2006;193:1333.

19. Cunliffe NA, Gondwe JS, Graham SM, Thindwa BD, Dove W, Broadhead RL, et al. Rotavirus strain diversity in Blantyre, Malawi, from 1997 to 1999. Journal of clinical microbiology.2001;39:836-843.

20. Ramshaw IA, Ramsay AJ, Karupiah G, Rolph MS, Mahalingam S, Ruby JC. Cytokines and immunity to viral infections. Immunol Rev.1997;159:119-135.

21. Dinarello CA. Proinflammatory cytokines. Chest.2000;118:503-508.

22. Azim T, Ahmad SM, Sefat EK, Sarker MS, Unicomb LE, De S, et al. Immune response of children who develop persistent diarrhoea following rotavirus infection. Clin Diagn Lab Immunol.1999;6:690-695.

23. Jiang B, Snipes-Magaldi L, Dennehy P, Keyserling H, Holman RC, Bresee J, et al. Cytokines as mediators for or effectors against rotavirus disease in children. Clin Diagn Lab Immunol.2003;10:995-1001. 